THE Astrophysical Journal, 285:515-526, 1984 October 15

(C) 1984. The American Astronomical Society. All rights reserved. Printed in U.S.A.

\title{
NEAR-INFRARED PHOTOMETRY OF FAINT RADIO GALAXIES IN SELECTED AREAS
}

\author{
TRINH X. THUAN ${ }^{1}$ \\ Astronomy Department, University of Virginia, Charlottesville \\ ROGIER A. WINDHORST ${ }^{1}$ \\ Sterrewacht, Leiden \\ JEFFERY J. PUSChELL ${ }^{1}$ \\ Titan Systems Inc., La Jolla \\ RICHARD B. ISAACMAN \\ United Kingdom Infrared Telescope, Hawaii \\ AND \\ Frazer N. OWEN ${ }^{1}$ \\ National Radio Astronomy Observatory, ${ }^{2}$ Soccoro, New Mexico \\ Received 1983 October 6; accepted 1984 May 1
}

\begin{abstract}
We present near-infrared $J H K$ photometry for 40 radio galaxies and 8 quasars from the complete sample of mJy radio sources in the Leiden-Berkeley Deep Survey. The detection rate is 48 out of 52 attempted objects down to $K \sim 17.5 \mathrm{mag}$. Combining these measurements with optical photometry and spectral evolution models, the following conclusions are derived. The optical-infrared colors of faint radio galaxies are independent of their radio flux and power and are due to their stellar populations. The fraction of very red $\mathrm{mJy}$ radio galaxies, with nonthermal infrared emission, is small $(\$ 10 \%)$. The colors of about two-thirds of the mJy radio galaxies are consistent with those of distant $(z \gtrsim 0.2)$ nonevolving or mildly evolving luminous giant elliptical galaxies $\left(M_{v}=-23, H_{0}=50 \mathrm{~km} \mathrm{~s}^{-1} \mathrm{Mpc}^{-1}, q_{0}=0\right)$. The remaining third of the mJy galaxies are bluer than elliptical galaxy models. The available redshift information appears to indicate that they may be nearer $(z \lesssim$ $0.3)$ galaxies of lower luminosity $\left(M_{v} \gtrsim-22\right)$ undergoing star formation.
\end{abstract}

Subject headings: galaxies: photometry — infrared: sources — quasars — radio sources: galaxies

\section{INTRODUCTION}

Over the past few years, very deep radio surveys at 1412 $\mathrm{MHz}$ have been carried out with the $3 \mathrm{~km}$ Westerbork Synthesis Radio Telescope in the selected areas SA 57, SA 68, and the Hercules and Lynx fields, covering a total area of 5.52 square degrees and yielding a complete sample of 306 radio sources with $S_{1412 \mathrm{MHz}} \geq 0.6 \mathrm{mJy}$ (Windhorst, van Heerde, and Katgert 1984). Deep multicolor prime-focus Kitt Peak $4 \mathrm{~m}$ plates have also been obtained for these fields in the passbands $U^{+}$(IIIa-J plate + UG 5 filter), $J^{+}$(IIIa-J + GG 385), $F(127-02+$ GG 495), and $N$ (IV-N + RG 695). We denote here by $U^{+}$and $J^{+}$ the original $U$ and $J$ bands of Kron (1980) and Koo (1981) in order to avoid confusion with the Johnson (1966) $U$ band and the infrared $J$ band at $1.24 \mu \mathrm{m}$. More than $50 \%$ of the radio sources in the complete sample, hereafter called the LeidenBerkeley Deep Survey (LBDS) sources, have been optically identified, about $80 \%$ of the optical identifications being faint $(V \geq 21.5 \mathrm{mag})$ galaxies. The optical identifications are complete down to $\sim 23.3 \mathrm{mag}$ in $U^{+}\left(\lambda_{\text {eff }} \sim 3600 \AA\right), 23.7 \mathrm{mag}$ in $\left.J^{+} \lambda_{\text {eff }} \sim 4650 \AA\right), 22.7 \mathrm{mag}$ in $F\left(\lambda_{\text {eff }} \sim 6100 \AA\right)$, and $21.1 \mathrm{mag}$ in $N\left(\lambda_{\text {eff }} \sim 8000 \AA\right)$ (Windhorst, Kron, and Koo 1984).

We present in this paper the results of a program of nearinfrared measurements of a considerable subsample of the

\footnotetext{
${ }^{1}$ Visiting Astronomer at the Infrared Telescope Facility, which is operated by the University of Hawaii under contract with the National Aeronautics and Space Administration.

${ }^{2}$ The National Radio Astronomy Observatory is operated by the Associated Universities, Inc., under contract with the National Science Foundation.
}

LBDS sources. There are several reasons for undertaking such a program.

1. The LBDS sources have radio fluxes in the mJy range. They are more than 3 orders of magnitude fainter in radio than the much-studied $3 \mathrm{CR}$ radio sources and thus may be more representative of the typical radio galaxy. Their infrared study is complementary to that of the $3 \mathrm{C}$ radio sources (Lilly and Longair 1982; Puschell, Owen, and Laing 1982) and that of optically selected radio-quiet galaxies (Ellis and Allen 1983). By combining the infrared results with optical measurements, a comparison of the stellar populations in these three types of galaxies can be made.

2. Radio-bright $3 \mathrm{CR}$ sources are known to be associated with luminous giant elliptical radio galaxies and quasars. The faint LBDS radio sources are associated with a wider range of galaxy morphological types, including not only giant elliptical but also spiral galaxies of lower luminosity. The 3CR radio sources appear to have optical-infrared colors bluer than those predicted by a redshifted nonevolving elliptical spectral energy distribution (SED), although there is some controversy on the implied degree of color evolution in the near-infrared (Lebofsky 1981; Lilly and Longair 1982; Puschell, Owen, and Laing 1982). Do the infrared and optical-infrared colors of the LBDS also show evidence for evolution of the SED? If so, how does the color evolution of the mJy radio galaxies compare with that suggested for the 3CR sources (Lilly and Longair 1982) and for optically selected galaxies (Ellis and Allen 1983)?

3. Finally, Dressler and Gunn (1983), as a result of a spectroscopic study of one of the Butcher-Oemler (1978) clusters, 
TABLE 1

THE COMPLETE INFRARED SUBSAMPLE

\begin{tabular}{|c|c|c|c|c|c|c|c|c|}
\hline Name & $\begin{array}{l}\text { R.A.(1950) } \\
\text { Decl.(1950) }\end{array}$ & $\begin{array}{l}\text { Type } \\
\text { Env. }^{a}\end{array}$ & $z^{\mathrm{b}}$ & $\begin{array}{c}J(\mathrm{mag}) \\
F_{J}(\mathrm{mJy})\end{array}$ & $\begin{array}{c}H(\mathrm{mag}) \\
F_{H}(\mathrm{mJy})\end{array}$ & $\begin{array}{c}K(\mathrm{mag}) \\
F_{H}(\mathrm{mJy})\end{array}$ & $\begin{array}{c}\text { Date } \\
\text { Observed } \\
\text { (yr/mo/day) }\end{array}$ & Tel. $^{\mathrm{c}}$ \\
\hline \multicolumn{9}{|c|}{ Selected Area 57} \\
\hline $52 \mathrm{~W} 008 \ldots \ldots$ & $\begin{array}{r}130500.7 \\
+291827\end{array}$ & $\begin{array}{l}\mathrm{G} \\
\mathrm{cl}\end{array}$ & $\ldots$ & $\ldots$ & $\ldots$ & $\begin{array}{l}17.12 \pm 0.22 \\
0.092 \pm 0.017\end{array}$ & $82 / 06 / 14$ & $\mathrm{U}$ \\
\hline $52 \mathrm{~W} 013 \ldots \ldots$ & $\begin{array}{r}130526.3 \\
+294147\end{array}$ & $\begin{array}{l}\mathrm{GcD} \\
\mathrm{cl}\end{array}$ & 0.241 & $\begin{array}{r}15.88 \pm 0.08 \\
0.72 \pm 0.06\end{array}$ & $\begin{array}{r}14.92 \pm 0.07 \\
1.10 \pm 0.07\end{array}$ & $\begin{array}{r}14.33 \pm 0.07 \\
1.20 \pm 0.08\end{array}$ & $82 / 03 / 25$ & I \\
\hline $52 \mathrm{~W} 023 \ldots \ldots$ & $\begin{array}{r}130611.6 \\
+294226\end{array}$ & $\begin{array}{l}\text { Gc } \\
\mathrm{gr}\end{array}$ & 0.202 & $\ldots$ & $\ldots$ & $\begin{array}{l}17.31 \pm 0.25 \\
0.077 \pm 0.016\end{array}$ & $82 / 06 / 14$ & $\mathrm{U}$ \\
\hline $52 \mathrm{~W} 034 \ldots \ldots$ & $\begin{aligned} 130645.5 \\
+293004\end{aligned}$ & $\mathrm{G}$ & $\ldots$ & $\ldots$ & $\ldots$ & $\begin{array}{l}17.84 \pm 0.45 \\
0.048 \pm 0.016\end{array}$ & $82 / 06 / 14$ & $\mathrm{U}$ \\
\hline $52 \mathrm{~W} 044 \ldots \ldots$ & $\begin{array}{r}130714.6 \\
+294126\end{array}$ & Gc & 0.300 & $\begin{array}{r}17.31 \pm 0.26 \\
0.19 \pm 0.05\end{array}$ & $\cdots$ & $\begin{array}{r}15.28 \pm 0.12 \\
0.50 \pm 0.06\end{array}$ & $82 / 03 / 25$ & I \\
\hline
\end{tabular}

\begin{tabular}{|c|c|c|c|c|c|c|c|c|}
\hline \multicolumn{9}{|c|}{ Hercules } \\
\hline $53 \mathrm{~W} 005 \ldots \ldots$ & $\begin{array}{r}171322.4 \\
+503143\end{array}$ & $\begin{array}{l}\mathrm{G} \\
\mathrm{gr}\end{array}$ & .. & $\ldots$ & $\ldots$ & $\begin{array}{r}16.97 \pm 0.25 \\
0.11 \pm 0.02\end{array}$ & $82 / 06 / 14$ & $\mathrm{U}$ \\
\hline 53W $008 \ldots \ldots$ & $\begin{array}{r}171348.0 \\
+495736\end{array}$ & $\begin{array}{l}\mathrm{Q} \\
\mathrm{cl}\end{array}$ & $\ldots$ & $\begin{array}{r}17.68 \pm 0.28 \\
0.14 \pm 0.03\end{array}$ & $\ldots$ & $\begin{array}{r}16.57 \pm 0.21 \\
0.15 \pm 0.03\end{array}$ & $81 / 09 / 20$ & I \\
\hline $53 \mathrm{~W} 009 \ldots \ldots$ & $\begin{array}{r}171348.9 \\
+502449\end{array}$ & Q & $\ldots$ & $\begin{array}{r}16.42 \pm 0.10 \\
0.44 \pm 0.04\end{array}$ & $\begin{array}{r}16.12 \pm 0.11 \\
0.36 \pm 0.03\end{array}$ & $\begin{array}{r}15.15 \pm 0.11 \\
0.57 \pm 0.06\end{array}$ & $82 / 06 / 14$ & U \\
\hline $53 \mathrm{~W} 010 \ldots \ldots$ & $\begin{aligned} 171350.2 \\
+501338\end{aligned}$ & Gc & $\ldots$ & $\begin{array}{r}17.10 \pm 0.14 \\
0.23 \pm 0.03\end{array}$ & $\begin{array}{r}16.38 \pm 0.15 \\
0.28 \pm 0.04\end{array}$ & $\begin{array}{r}15.47 \pm 0.07 \\
0.42 \pm 0.03\end{array}$ & $82 / 06 / 14$ & $\mathrm{U}$ \\
\hline $53 \mathrm{~W} 020 \ldots \ldots$ & $\begin{array}{r}171434.2 \\
+502815\end{array}$ & $\begin{array}{l}\mathrm{GE} \\
\mathrm{gr}\end{array}$ & 0.100 & $\begin{array}{r}15.36 \pm 0.07 \\
1.15 \pm 0.07\end{array}$ & $\begin{array}{r}14.60 \pm 0.06 \\
1.47 \pm 0.08\end{array}$ & $\begin{array}{r}14.21 \pm \text { p.06 } \\
1.35 \pm 0.07\end{array}$ & $82 / 06 / 13$ & $\mathrm{U}$ \\
\hline $53 \mathrm{~W} 021 \ldots \ldots$ & $\begin{array}{r}171450.1 \\
+502356\end{array}$ & $\begin{array}{l}\text { Q? } \\
\text { gr }\end{array}$ & $\ldots$ & $\ldots$ & $\begin{array}{l}18.38 \pm 0.45 \\
0.045 \pm 0.015\end{array}$ & $\begin{array}{r}16.37 \pm 0.15 \\
0.18 \pm 0.02\end{array}$ & $82 / 06 / 14$ & $\mathrm{U}$ \\
\hline $53 \mathrm{~W} 023 \ldots$ & $\begin{array}{r}171455.0 \\
+500846\end{array}$ & $\mathrm{G}$ & $\ldots$ & $\begin{array}{r}17.87 \pm 0.54 \\
0.11 \pm 0.04\end{array}$ & $\ldots$ & $\begin{array}{r}15.54 \pm 0.16 \\
0.40 \pm 0.05\end{array}$ & $82 / 03 / 25$ & I \\
\hline $53 \mathrm{~W} 026 \ldots \ldots$ & $\begin{array}{r}171512.1 \\
+495024\end{array}$ & $\begin{array}{l}\mathrm{G} \\
\mathrm{gr}\end{array}$ & $\ldots$ & $\ldots$ & $\ldots$ & $\begin{array}{r}16.39 \pm 0.21 \\
0.18 \pm 0.03\end{array}$ & $82 / 06 / 14$ & $\mathrm{U}$ \\
\hline $53 \mathrm{~W} 031 \ldots \ldots$ & $\begin{array}{r}171530.7 \\
+495954\end{array}$ & G & 0.628 & $\begin{array}{r}17.02 \pm 0.16 \\
0.25 \pm 0.03\end{array}$ & $\begin{array}{r}16.58 \pm 0.13 \\
0.24 \pm 0.03\end{array}$ & $\begin{array}{r}15.72 \pm 0.11 \\
0.33 \pm 0.03\end{array}$ & $82 / 03 / 25$ & I \\
\hline $53 \mathrm{~W} 032$. & $\begin{array}{r}171531.1 \\
+495158\end{array}$ & GE & $\ldots$ & $\begin{array}{r}16.92 \pm 0.20 \\
0.27 \pm 0.05\end{array}$ & $\begin{array}{r}15.59 \pm 0.09 \\
0.59 \pm 0.05\end{array}$ & $\begin{array}{r}15.19 \pm 0.08 \\
0.55 \pm 0.04\end{array}$ & $82 / 06 / 14$ & $\mathrm{U}$ \\
\hline $53 \mathrm{~W} 039 \ldots \ldots$ & $\begin{array}{r}171547.5 \\
+502839\end{array}$ & $\begin{array}{l}\mathrm{GE} \\
\mathrm{cl}\end{array}$ & 0.402 & $\begin{array}{r}16.54 \pm 0.19 \\
0.39 \pm 0.06\end{array}$ & $\begin{array}{r}16.30 \pm 0.15 \\
0.31 \pm 0.04\end{array}$ & $\begin{array}{r}14.97 \pm 0.08 \\
0.67 \pm 0.05\end{array}$ & $82 / 06 / 14$ & $\mathrm{U}$ \\
\hline $53 \mathrm{~W} 044 \ldots \ldots$ & $\begin{array}{r}171621.5 \\
+500612\end{array}$ & GE & 0.311 & $\begin{array}{r}16.40 \pm 0.19 \\
0.44 \pm 0.07\end{array}$ & $\begin{array}{r}15.28 \pm 0.07 \\
0.79 \pm 0.05\end{array}$ & $\begin{array}{r}14.53 \pm 0.05 \\
1.20 \pm 0.05\end{array}$ & $82 / 06 / 14$ & $\mathrm{U}$ \\
\hline $53 \mathrm{~W} 045 \ldots \ldots$ & $\begin{array}{r}171626.7 \\
+501851\end{array}$ & Gc & $\ldots$ & $\begin{array}{r}17.67 \pm 0.29 \\
0.14 \pm 0.03\end{array}$ & $\ldots$ & $\begin{array}{r}15.59 \pm 0.19 \\
0.38 \pm 0.06\end{array}$ & $82 / 03 / 25$ & I \\
\hline $53 \mathrm{~W} 046 \ldots \ldots$ & $\begin{array}{r}171638.3 \\
+501057\end{array}$ & G & 0.545 & $\begin{array}{r}17.83 \pm 0.42 \\
0.12 \pm 0.05\end{array}$ & $\ldots$ & $\begin{array}{r}15.96 \pm 0.22 \\
0.27 \pm 0.06\end{array}$ & $82 / 03 / 25$ & I \\
\hline $53 \mathrm{~W} 047 \ldots \ldots$ & $\begin{array}{r}171653.5 \\
+502549\end{array}$ & $\begin{array}{l}\text { Gc } \\
d b\end{array}$ & $\ldots$ & $\ldots$ & $\ldots$ & $\begin{array}{l}17.67 \pm 0.36 \\
0.056 \pm 0.016\end{array}$ & $82 / 06 / 14$ & $\mathrm{U}$ \\
\hline $53 \mathrm{~W} 052 \ldots \ldots$ & $\begin{array}{r}171718.7 \\
+500154\end{array}$ & $\begin{array}{l}\mathrm{G} \\
\mathrm{gr}\end{array}$ & $\ldots$ & $\ldots$ & $\begin{array}{l}17.85 \pm 0.32 \\
0.074 \pm 0.019\end{array}$ & $\begin{array}{r}16.90 \pm 0.16 \\
0.11 \pm 0.02\end{array}$ & $82 / 06 / 13$ & $\mathrm{U}$ \\
\hline $53 \mathrm{~W} 061 \ldots \ldots$ & $\begin{array}{r}171811.3 \\
+494659\end{array}$ & $\mathrm{Q}$ ? & $\ldots$ & $\ldots$ & $\ldots$ & $\begin{array}{l}17.39 \pm 0.26 \\
0.072 \pm 0.015\end{array}$ & $82 / 06 / 13$ & $\mathrm{U}$ \\
\hline $53 \mathrm{~W} 062 \ldots \ldots$ & $\begin{array}{r}171816.6 \\
+500205\end{array}$ & $\begin{array}{l}\mathrm{G} \\
\mathrm{cl}\end{array}$ & $\ldots$ & $\ldots$ & $\ldots$ & $\begin{array}{l}17.09 \pm 0.25 \\
0.095 \pm 0.020\end{array}$ & $82 / 06 / 13$ & $\mathbf{U}$ \\
\hline 53W $075 \ldots \ldots$ & $\begin{array}{r}171926.4 \\
+494643\end{array}$ & $\mathrm{Q}$ & $\ldots$ & $\ldots$ & $\begin{array}{l}18.14 \pm 0.37 \\
0.057 \pm 0.016\end{array}$ & $\begin{array}{c}16.74 \pm 0.23 \\
0.13 \pm 0.03\end{array}$ & $82 / 06 / 13$ & $\mathrm{U}$ \\
\hline $53 \mathrm{~W} 076 \ldots \ldots$ & $\begin{array}{r}171939.8 \\
+494255\end{array}$ & Gc & 0.390 & $\begin{array}{r}16.77 \pm 0.11 \\
0.32 \pm 0.03\end{array}$ & $\begin{array}{r}16.13 \pm 0.11 \\
0.36 \pm 0.03\end{array}$ & $\begin{array}{r}15.56 \pm 0.10 \\
0.39 \pm 0.03\end{array}$ & $82 / 06 / 13$ & $\mathrm{U}$ \\
\hline $53 \mathrm{~W} 078 \ldots \ldots$ & $\begin{array}{r}172003.1 \\
+500626\end{array}$ & $\begin{array}{l}\mathrm{G} \\
\mathrm{gr}\end{array}$ & $\ldots$ & $\begin{array}{r}16.25 \pm 0.09 \\
0.51 \pm 0.04\end{array}$ & $\begin{array}{r}15.55 \pm 0.11 \\
0.61 \pm 0.07\end{array}$ & $\begin{array}{r}14.91 \pm 0.06 \\
0.71 \pm 0.04\end{array}$ & $82 / 06 / 13$ & $\mathrm{U}$ \\
\hline $53 \mathrm{~W} 080 \ldots \ldots$ & $\begin{array}{r}172022.1 \\
+495827\end{array}$ & Q & $\ldots$ & $\begin{array}{r}16.72 \pm 0.06 \\
0.33 \pm 0.02\end{array}$ & $\begin{array}{r}15.84 \pm 0.10 \\
0.47 \pm 0.04\end{array}$ & $\begin{array}{r}15.02 \pm 0.09 \\
0.64 \pm 0.05\end{array}$ & $82 / 06 / 13$ & $\mathrm{U}$ \\
\hline $53 \mathrm{~W} 085 \ldots \ldots$ & $\begin{array}{r}172037.1 \\
+495723\end{array}$ & $\mathrm{Q}$ ? & $\ldots$ & $\begin{array}{l}>18.2 \\
<0.084\end{array}$ & $\ldots$ & $\begin{array}{r}16.87 \pm 0.16 \\
0.12 \pm 0.02\end{array}$ & $82 / 06 / 13$ & $\mathrm{U}$ \\
\hline $53 \mathrm{~W} 086 \ldots \ldots$ & $\begin{array}{r}172042.2 \\
+495622\end{array}$ & G & $\ldots$ & $\ldots$ & $\begin{array}{r}17.29 \pm 0.21 \\
0.12 \pm 0.02\end{array}$ & $\begin{array}{r}16.41 \pm 0.10 \\
0.18 \pm 0.02\end{array}$ & $82 / 06 / 13$ & $\mathrm{U}$ \\
\hline
\end{tabular}

(continued) 
TABLE 1 -Continued

\begin{tabular}{|c|c|c|c|c|c|c|c|c|}
\hline Name & $\begin{array}{l}\text { R.A.(1950) } \\
\text { Eecl.(1950) }\end{array}$ & $\begin{array}{l}\text { Type } \\
\text { Env. }\end{array}$ & $z^{\mathrm{b}}$ & $\begin{array}{c}J(\mathrm{mag}) \\
F_{J}(\mathrm{mJy})\end{array}$ & $\begin{array}{c}H(\mathrm{mag}) \\
F_{H}(\mathrm{mJy})\end{array}$ & $\begin{array}{c}K(\mathrm{mag}) \\
F_{H}(\mathrm{mJy})\end{array}$ & $\begin{array}{c}\text { Date } \\
\text { Observed } \\
\text { (yr/mo/day) }\end{array}$ & Tel..$^{\mathrm{c}}$ \\
\hline \multicolumn{9}{|c|}{ Selected Area 68} \\
\hline $54 \mathrm{~W} 009^{d} \ldots$. & $\begin{array}{r}001320.7 \\
+155818\end{array}$ & $\begin{array}{l}\mathrm{GcD} \\
\mathrm{gr}\end{array}$ & 0.083 & $\begin{array}{r}13.78 \pm 0.03 \\
4.96 \pm 0.12\end{array}$ & $\begin{array}{c}12.89 \pm 0.02 \\
7.11 \pm 0.1\end{array}$ & $\begin{array}{r}12.44 \pm 0.01 \\
6.84 \pm 0.08\end{array}$ & $81 / 09 / 19$ & I \\
\hline $54 \mathrm{~W} 013 \ldots \ldots$ & $\begin{array}{r}001339.8 \\
+161129\end{array}$ & $\mathrm{Gc}$ & 0.249 & $\begin{array}{r}16.36 \pm 0.06 \\
0.46 \pm 0.03\end{array}$ & $\begin{array}{r}15.32 \pm 0.05 \\
0.76 \pm 0.04\end{array}$ & $\begin{array}{r}13.95 \pm 0.05 \\
1.70 \pm 0.08\end{array}$ & $81 / 09 / 19$ & I \\
\hline $54 \mathrm{~W} 017 \ldots \ldots$ & $\begin{array}{r}001349.3 \\
+154144\end{array}$ & $\begin{array}{l}\mathrm{Gc} \\
\mathrm{cl}\end{array}$ & 0.512 & $\ldots$ & $\ldots$ & $\begin{array}{r}17.01 \pm 0.29 \\
0.10 \pm 0.03\end{array}$ & $81 / 09 / 21$ & I \\
\hline $54 \mathrm{~W} 021 \ldots \ldots$ & $\begin{array}{r}001352.4 \\
+160422\end{array}$ & $\begin{array}{l}\mathrm{Gc} \\
\mathrm{gr}\end{array}$ & $\ldots$ & $\begin{array}{r}17.71 \pm 0.15 \\
0.13 \pm 0.02\end{array}$ & $\begin{array}{r}16.82 \pm 0.15 \\
0.19 \pm 0.02\end{array}$ & $\begin{array}{r}15.91 \pm 0.09 \\
0.28 \pm 0.02\end{array}$ & $82 / 10 / 16$ & I \\
\hline $54 \mathrm{~W} 028 \ldots \ldots$ & $\begin{array}{r}001406.0 \\
+155152\end{array}$ & GE & 0.173 & $\begin{array}{r}15.75 \pm 0.04 \\
0.81 \pm 0.03\end{array}$ & $\begin{array}{r}14.97 \pm 0.05 \\
1.05 \pm 0.04\end{array}$ & $\begin{array}{r}14.39 \pm 0.04 \\
1.14 \pm 0.04\end{array}$ & $81 / 09 / 19$ & I \\
\hline $54 \mathrm{~W} 036 \ldots \ldots$ & $\begin{array}{r}001426.1 \\
+152254\end{array}$ & G & $\cdots$ & $\begin{array}{r}>18.13 \\
<0.09\end{array}$ & $\ldots$ & $\begin{array}{r}16.72 \pm 0.22 \\
0.13 \pm 0.03\end{array}$ & $81 / 09 / 20$ & I \\
\hline $54 \mathrm{~W} 038 \ldots \ldots$ & $\begin{array}{r}001428.5 \\
+155400\end{array}$ & $\mathrm{G}$ & $\ldots$ & $\begin{array}{r}17.91 \pm 0.20 \\
0.11 \pm 0.02\end{array}$ & $\ldots$ & $\begin{array}{r}16.50 \pm 0.17 \\
0.16 \pm 0.03\end{array}$ & $81 / 09 / 20$ & I \\
\hline $54 \mathrm{~W} 039 \ldots \ldots$ & $\begin{array}{r}001429.2 \\
+152635\end{array}$ & $\mathrm{G}$ & $\cdots$ & $\begin{array}{l}18.54 \pm 0.20 \\
0.062 \pm 0.010\end{array}$ & $\cdots$ & $\begin{array}{r}16.48 \pm 0.13 \\
0.17 \pm 0.02\end{array}$ & $82 / 10 / 16$ & I \\
\hline $54 \mathrm{~W} 050 \ldots \ldots$ & $\begin{array}{r}001500.3 \\
+151248\end{array}$ & $\begin{array}{l}\mathrm{G} \\
\mathrm{cl}\end{array}$ & $\ldots$ & $\ldots$ & $\cdots$ & $\begin{array}{r}17.10 \pm 0.24 \\
0.09 \pm 0.02\end{array}$ & $81 / 09 / 20$ & I \\
\hline $54 \mathrm{~W} 058 \ldots \ldots$ & $\begin{array}{r}001514.5 \\
+152305\end{array}$ & $\mathrm{G}$ & $\ldots$ & $\begin{array}{l}18.31 \pm 0.22 \\
0.076 \pm 0.014\end{array}$ & $\ldots$ & $\begin{array}{r}16.28 \pm 0.12 \\
0.20 \pm 0.02\end{array}$ & $82 / 10 / 16$ & I \\
\hline $54 \mathrm{~W} 072 \ldots \ldots$ & $\begin{array}{r}001542.3 \\
+162645\end{array}$ & GS & $\ldots$ & $\begin{array}{c}16.91 \pm 0.017 \\
0.28 \pm 0.04\end{array}$ & $\begin{array}{r}15.87 \pm 0.10 \\
0.46 \pm 0.04\end{array}$ & $\begin{array}{r}15.17 \pm 0.08 \\
0.56 \pm 0.04\end{array}$ & $82 / 10 / 17$ & I \\
\hline $54 \mathrm{~W} 073 \ldots \ldots$ & $\begin{array}{r}001549.0 \\
+152339\end{array}$ & $\begin{array}{l}\mathrm{Q} \\
\mathrm{gr}\end{array}$ & $\ldots$ & $\begin{array}{r}18.06 \pm 0.16 \\
0.09 \pm 0.02\end{array}$ & $\begin{array}{r}17.45 \pm 0.24 \\
0.11 \pm 0.03\end{array}$ & $\begin{array}{r}16.87 \pm 0.23 \\
0.12 \pm 0.03\end{array}$ & $81 / 09 / 20$ & I \\
\hline $54 \mathrm{~W} 078 \ldots \ldots$ & $\begin{array}{r}001553.8 \\
+151402\end{array}$ & $\begin{array}{l}\mathrm{Gc} \\
\mathrm{cl}\end{array}$ & $\cdots$ & $\begin{array}{r}17.31 \pm 0.14 \\
0.19 \pm 0.02\end{array}$ & $\begin{array}{r}16.39 \pm 0.20 \\
0.28 \pm 0.05\end{array}$ & $\begin{array}{r}15.12 \pm 0.10 \\
0.58 \pm 0.05\end{array}$ & $82 / 10 / 17$ & I \\
\hline $54 \mathrm{~W} 081 \ldots \ldots$ & $\begin{array}{r}001601.9 \\
+514151\end{array}$ & $\mathrm{G}$ & $\ldots$ & $\ldots$ & $\ldots$ & $\begin{array}{r}16.30 \pm 0.17 \\
0.20 \pm 0.03\end{array}$ & $82 / 10 / 17$ & I \\
\hline $54 \mathrm{~W} 084 \ldots \ldots$ & $\begin{array}{r}001606.2 \\
+154530\end{array}$ & Gc & $\ldots$ & $\begin{array}{r}16.84 \pm 0.15 \\
0.30 \pm 0.04\end{array}$ & $\begin{array}{r}16.22 \pm 0.10 \\
0.33 \pm 0.03\end{array}$ & $\begin{array}{r}15.31 \pm 0.07 \\
0.49 \pm 0.03\end{array}$ & $82 / 10 / 16$ & I \\
\hline $54 \mathrm{~W} 088 \ldots \ldots$ & $\begin{array}{r}001635.0 \\
+153543 \\
\end{array}$ & G & $\ldots$ & $\begin{array}{r}16.96 \pm 0.18 \\
0.26 \pm 0.05\end{array}$ & $\begin{array}{r}16.33 \pm 0.18 \\
0.30 \pm 0.05 \\
\end{array}$ & $\begin{array}{r}15.51 \pm 0.11 \\
0.40 \pm 0.04 \\
\end{array}$ & $81 / 09 / 21$ & I \\
\hline & & & & Lynx & & & & \\
\hline $55 \mathrm{~W} 019 \ldots \ldots$ & $\begin{aligned} 083341.8 \\
+451243\end{aligned}$ & $\begin{array}{l}\mathrm{G} \\
\mathrm{gr}\end{array}$ & $\cdots$ & $\ldots$ & $\ldots$ & $\begin{array}{l}17.11 \pm 0.41 \\
0.092 \pm 0.042\end{array}$ & $82 / 03 / 25$ & I \\
\hline $55 \mathrm{~W} 037 \ldots \ldots$ & $\begin{array}{r}083427.3 \\
+450057\end{array}$ & GE & 0.210 & $\begin{array}{r}15.70 \pm 0.09 \\
0.85 \pm 0.07\end{array}$ & $\begin{array}{r}14.84 \pm 0.07 \\
1.19 \pm 0.08\end{array}$ & $\begin{array}{r}14.19 \pm 0.05 \\
1.36 \pm 0.07\end{array}$ & $82 / 03 / 25$ & $\mathbf{I}$ \\
\hline $55 \mathrm{~W} 063 \ldots \ldots$ & $\begin{array}{r}083636.8 \\
+445530\end{array}$ & $\begin{array}{l}\text { GcD } \\
\mathrm{cl}\end{array}$ & $\ldots$ & $\begin{array}{r}16.28 \pm 0.11 \\
0.49 \pm 0.05\end{array}$ & $\begin{array}{r}15.48 \pm 0.09 \\
0.65 \pm 0.06\end{array}$ & $\begin{array}{r}14.82 \pm 0.09 \\
0.76 \pm 0.07\end{array}$ & $82 / 03 / 25$ & I \\
\hline
\end{tabular}

a Type: $\mathrm{G}=$ galaxy, $\mathrm{Q}=$ quasar, ? = uncertain, $\mathrm{E}=$ elliptical, $\mathrm{S}=$ spiral, $\mathrm{c}=$ compact. Environment: $\mathrm{cD}=\mathrm{cD}$ galaxy, $\mathrm{db}=$ double (nucleus), $\mathrm{gr}=$ in group, $\mathrm{cl}=$ in cluster.

b The redshifts are from Kron, Koo, and Windhorst 1984 and are accurate to 0.01 .

c $\mathrm{I}=3 \mathrm{~m}$ IRTF telescope; $\mathrm{U}=3.8 \mathrm{~m}$ UKIRT telescope.

${ }^{\mathrm{d}}$ For $54 \mathrm{~W} 009$ we also have a $3.4 \mu \mathrm{m}$ measurement: $L=12.45 \pm 0.26 \mathrm{mag}\left(F_{L}=3.14 \pm 0.86 \mathrm{mJy}\right)$.

have suggested that there may also be an increase in nonthermal activity in certain types of galaxies at times earlier than $\sim 5 \times 10^{9}$ years ago. Nonthermal emitters such as Seyfert or $\mathrm{N}$ galaxies usually show strong infrared excesses at $2.2 \mu \mathrm{m}$, so that near-infrared photometry provides a convenient way to detect nonthermal activity at large redshifts.

The observations are described in $\S$ II. We combine the optical and infrared measurements and compare the observations with galactic evolution models in $\S$ III. Our conclusions are summarized in $\S$ IV.

\section{OBSERVATIONS}

The observations were obtained with the $3 \mathrm{~m}$ NASA Infrared Telescope Facility (IRTF) during the periods 1981 September 17-19, 1982 March 22-24, and 1982 October 16-17, and with the $3.8 \mathrm{~m}$ United Kingdom Infrared Telescope (UKIRT) during the periods 1981 September 19-22 and 1982 June 11-14. The observation dates for individual galaxies are given in Table 1. On both telescopes, InSb photometers cooled with liquid nitrogen were used. A 7".8 aperture was used at the IRTF. At UKIRT, a 7".5 aperture was used for the majority of the observations except for a few galaxies in dense clusters, where a $5^{\prime \prime}$ aperture was used, and for very bright galaxies $\left(V \lesssim 19 \mathrm{mag}\right.$ ), for which a $12^{\prime \prime}$ aperture was used. Subtraction of the sky background was done by on-off chopping with a wobbling secondary mirror. A north-south beam throw varying between $20^{\prime \prime}$ and $40^{\prime \prime}$ was used at the IRTF, and a $30^{\prime \prime}-60^{\prime \prime}$ east-west beam throw was used at UKIRT. In all cases, the beam throw was larger than the optical size of the observed objects.

The radio sources have positional accuracies between 0.5 and 2 ".5. The optical identification positions derived from astrometry on the $4 \mathrm{~m}$ plates have a typical accuracy of $\sim 0$ ". 4 . The telescope was offset to the optical position from stars bright enough to be seen on the TV monitor and $\sim 2$ ' away. The positions of these stars were measured on copies of the 
blue plates of the Palomar Sky Survey to an accuracy of 0 " 3 . For each galaxy the accuracy of the telescope setting was checked by first offsetting to a relatively bright star $\sim 2^{\prime}$ away from the radio galaxy's position. In all cases, the offset procedure yielded telescope settings accurate to better than 1 ".

Standard stars taken from the list of Elias et al. (1982) were measured frequently throughout the night to accurately determine the air-mass extinction corrections. In general, the extinction is less than 0.10 mag per unit air mass in the three bands $J(1.24 \mu \mathrm{m}), H(1.65 \mu \mathrm{m})$ and $K(2.22 \mu \mathrm{m})$. A high extinction of 0.4 mag per unit air mass was observed in $J$, and 0.3 mag per unit air mass in $H$ and $K$ during the UKIRT run in 1982 June. Despite the large extinction, the regression fits to the air-mass corrections show rms deviations of only $0.03-0.04 \mathrm{mag}$. These errors were added in quadrature to the statistical photoncounting errors.

In total, 52 identified radio sources were observed from the complete sample of Windhorst, Kron, and Koo (1984), which includes all LBDS radio sources with a peak radio signal-tonoise ratio in excess of 5 and a reliable optical identification on at least two of the $U^{+} J^{+} F N 4$ m prime-focus plates down to the magnitude limits mentioned in $\S \mathrm{I}$, a total of 171 objects. Another six near-infrared detections were made for objects not in the complete sample. Preference was given to galaxy identifications ( $\sim 80 \%$ of all objects), although eight possible quasars were also observed. Of these, three are not classified with certainty as stellar.

The usual observing procedure is to start with filter $K$ and to go on to filters $J$ and $H$ only if the candidate object is detected in $K$ with a signal-to-noise ratio larger than $\sim 5$. For the very faint objects, integrations are continued until at least the $3 \sigma$ level is reached. The near-infrared observations corrected for galactic extinction are given in Table 1 . The reddening corrections were calculated using the polar cap model of Sandage
(1973) and van de Hulst's reddening curve No. 15 (Johnson 1968), so that

$$
\begin{aligned}
& A_{i}=a_{i}\left(\csc \left|b^{\mathrm{II}}\right|-1\right) \quad \text { for } \quad\left|b^{\mathrm{II}}\right| \leq 50^{\circ} \\
& =0 \quad \text { for } \quad\left|b^{\mathrm{II}}\right|>50^{\circ} \text {, }
\end{aligned}
$$

where $i$ denotes the infrared bands $J, H, K$, and $L ; a_{J}=0.025$ mag, $a_{H}=0.015 \mathrm{mag}, a_{K}=0.009 \mathrm{mag}$, and $a_{L}=0.005 \mathrm{mag}$; and $b^{\mathrm{II}}$ is the galactic latitude. The corrections are very small, less than $0.02 \mathrm{mag}$ at $J$ and much smaller at longer wavelengths. The magnitude-to-flux calibrations are obtained by convolving the atmosphere-corrected filter responses with the model for $\alpha$ Lyrae given by Schild, Peterson, and Oke (1971) and normalizing to the $0.56 \mu \mathrm{m}$ flux of Oke and Schild (1970). The calibration gives $F_{J}=1.61 \mathrm{mJy}$ at $1.24 \mu \mathrm{m}, F_{H}=1.02 \mathrm{mJy}$ at $1.65 \mu \mathrm{m}, F_{K}=0.65 \mathrm{mJy}$ at $2.22 \mu \mathrm{m}$, and $F_{L}=0.30 \mathrm{mJy}$ at $3.40 \mu \mathrm{m}$ for a 15 th-magnitude star (Puschell, Owen, and Laing 1982). A few objects were observed both at the IRTF and at the UKIRT to check the calibration scales. The results were consistent within the errors.

We detected 48 objects out of a total of 52 observed sources in the complete sample, or $92 \%$. This high detection rate demonstrates the power of near-infrared techniques for the study of distant galaxies near the plate limit of the largest existing optical reflectors ( $B \sim 24 \mathrm{mag}$ ). Table 2 gives upper limits in $K$ for objects that were not detected. Upper limits are also given for some additional objects not in the complete sample of Windhorst, Kron, and Koo (1984).

The $U^{+} J^{+} F N$ optical magnitudes for the LBDS objects have been derived by photoelectrically calibrated photometry on Mayall $4 \mathrm{~m}$ plates (Kron, Koo, and Windhorst 1984). The errors in the optical magnitudes vary from $\sim 0.05$ mag for the brighter objects $\left(J^{+}<21.5 \mathrm{mag}\right)$ to $\sim 0.3 \mathrm{mag}$ for objects at the plate limit $\left(J^{+} \sim 24 \mathrm{mag}\right)$. The circular aperture of $7^{\prime \prime} .5$ in

TABLE 2

\begin{tabular}{|c|c|c|c|c|c|c|c|}
\hline Name & $\begin{array}{l}\text { R.A.(1950) } \\
\text { Decl.(1950) }\end{array}$ & $\begin{array}{l}\text { Type } \\
\text { Env. }^{a}\end{array}$ & $\begin{array}{c}J(\mathrm{mag}) \\
F_{J}(\mathrm{mJy})\end{array}$ & $\begin{array}{c}H(\mathrm{mag}) \\
F_{H}(\mathrm{mJy})\end{array}$ & $\begin{array}{l}K(\mathrm{mag}) \\
F_{K}(\mathrm{mJy})\end{array}$ & $\begin{array}{c}\text { Date } \\
\text { Observed } \\
\text { (yr/mo/day) }\end{array}$ & Tel. $^{\text {b }}$ \\
\hline $52 \mathrm{~W} 019^{\mathrm{c}}$ & $\begin{array}{r}130557.4 \\
+292223\end{array}$ & $\begin{array}{l}\mathrm{G} \\
\mathrm{gr}\end{array}$ & $\ldots$ & $\ldots$ & $\begin{array}{l}16.55 \pm 0.48 \\
0.160 \pm 0.056\end{array}$ & $82 / 06 / 14$ & $\mathrm{U}$ \\
\hline $53 \mathrm{~W} 028^{\mathrm{d}}$ & $\begin{aligned} 171513.4 \\
+503947\end{aligned}$ & $\mathrm{G}$ & $\begin{array}{r}16.41 \pm 0.15 \\
0.44 \pm 0.06\end{array}$ & $\begin{array}{r}15.66 \pm 0.11 \\
0.56 \pm 0.05\end{array}$ & $\begin{array}{r}14.97 \pm 0.06 \\
0.67 \pm 0.04\end{array}$ & $82 / 06 / 14$ & $\mathrm{U}$ \\
\hline $54 \mathrm{~W} 005^{\mathrm{d}} .$. & $\begin{array}{r}001252.9 \\
+163328\end{array}$ & $\mathrm{G} ?$ & $\begin{array}{r}15.50 \pm 0.06 \\
1.02 \pm 0.05\end{array}$ & $\begin{array}{r}14.55 \pm 0.06 \\
1.54 \pm 0.08\end{array}$ & $\begin{array}{r}13.93 \pm 0.04 \\
1.74 \pm 0.06\end{array}$ & $82 / 10 / 17$ & I \\
\hline $54 \mathrm{~W} 014^{\mathrm{e}} \ldots \ldots \ldots$ & $\begin{array}{r}001340.2 \\
+153441\end{array}$ & $?$ & $\ldots$ & $\ldots$ & $\begin{array}{l}>17.08 \\
<0.096\end{array}$ & $82 / 10 / 16$ & I \\
\hline $54 \mathrm{~W} 047^{\mathrm{d}} \ldots \ldots$ & $\begin{aligned} 001453.0 \\
+162314\end{aligned}$ & Q & $\ldots$ & $\ldots$ & $\begin{array}{r}16.96 \pm 0.20 \\
0.11 \pm 0.22\end{array}$ & $82 / 10 / 17$ & I \\
\hline $54 \mathrm{~W} 048^{\mathrm{e}} \ldots \ldots \ldots$ & $\begin{array}{r}001455.5 \\
+163222\end{array}$ & Q & $\ldots$ & $\ldots$ & $\begin{array}{l}>17.28 \\
<0.080\end{array}$ & $82 / 10 / 17$ & I \\
\hline $54 \mathrm{~W} 053^{\mathrm{e}} \ldots \ldots \ldots$ & $\begin{array}{r}001506.1 \\
+153056\end{array}$ & $\begin{array}{l}\mathrm{G} \\
\mathrm{db}\end{array}$ & $\cdots$ & $\ldots$ & $\begin{array}{l}>17.34 \\
<0.075\end{array}$ & $82 / 10 / 17$ & I \\
\hline $54 \mathrm{~W} 056^{\mathrm{c}} \ldots$ & $\begin{array}{r}001509.0 \\
+153729\end{array}$ & $\begin{array}{l}\mathrm{G} \\
\mathrm{gr}\end{array}$ & $\begin{array}{r}17.41 \pm 0.18 \\
0.17 \pm 0.03\end{array}$ & $\begin{array}{r}16.15 \pm 0.11 \\
0.35 \pm 0.03\end{array}$ & $\begin{array}{r}15.57 \pm 0.14 \\
0.38 \pm 0.05\end{array}$ & $82 / 10 / 17$ & I \\
\hline $54 \mathrm{~W} 070^{\mathrm{e}} \ldots \ldots \ldots$ & $\begin{array}{r}001541.2 \\
+155706\end{array}$ & $\mathrm{Q}$ ? & $\ldots$ & $\ldots$ & $\begin{array}{l}>17.13 \\
<0.091\end{array}$ & $82 / 10 / 17$ & I \\
\hline $54 \mathrm{~W} 089^{d}$ & $\begin{aligned} 001652.9 \\
+153915\end{aligned}$ & Q & $\ldots$ & $\ldots$ & $\begin{array}{l}17.32 \pm 0.34 \\
0.077 \pm 0.021\end{array}$ & $82 / 10 / 17$ & I \\
\hline
\end{tabular}

AdDitional Observations

${ }^{\text {a }}$ See Table 1 , note a.

${ }^{b}$ See Table 1 , note c.

${ }^{\mathrm{c}}$ Not in complete optical sample, but a possible identification.

${ }^{d}$ Not in complete radio sample (i.e., $4 \sigma \leq$ peak signal-to-noise ratio $<5 \sigma$ ).

${ }^{\mathrm{e}}$ In complete radio-optical sample, but not detected in $K$. 
the optical photometry is virtually identical with the aperture that we used for the infrared photometry of most of the objects.

Figure 1 shows the distribution in $J^{+}$(not corrected for galactic extinction) for the objects for which we have obtained infrared observations (dashed line) as compared with that for the complete sample (solid line). We have not included objects in the Lynx field because we have only three infrared observations out of a total of 85 galaxies, and their inclusion would bias the statistics. The arrow at $J^{+} \sim 23.7 \mathrm{mag}$ denotes the plate limit for complete optical identifications. It is clear from Figure 1 that for $J^{+} \lesssim 23 \mathrm{mag}$, the $J^{+}$distribution of the infrared subsample is similar to that of the original complete sample and that there is no evident selection effect. The infrared subsample is not in proportion to the optical sample for 23 mag $\lesssim J^{+} \lesssim 23.7 \mathrm{mag}$, although the few observed objects in this interval should be representative.

Since one of the aims of the paper is to compare the optical and infrared properties of strong and weak radio sources and since $V$ - and $R$-magnitudes are already available for $3 \mathrm{C}$ radio sources from Lilly and Longair (1982), it is useful to derive $V$ and $R$-magnitudes from the measured $J^{+} F N$ magnitudes for the LBDS sources. We have used the transformation equations given by Kron (1980) to derive

$$
V=F+0.34\left(J^{+}-F\right) \quad \text { and } \quad R=\frac{2 F+N}{3},
$$

which are accurate to $\sim 0.2 \mathrm{mag}$. The optical magnitudes were corrected for galactic extinction in the same manner as the infrared magnitudes, with $a_{V}=0.099 \mathrm{mag}$ and $a_{R}=0.071 \mathrm{mag}$. We also use the relation $B=1.20 J^{+}-0.19 F$ to derive $B$ magnitudes, with extinction coefficient $a_{B}=0.132 \mathrm{mag}$.

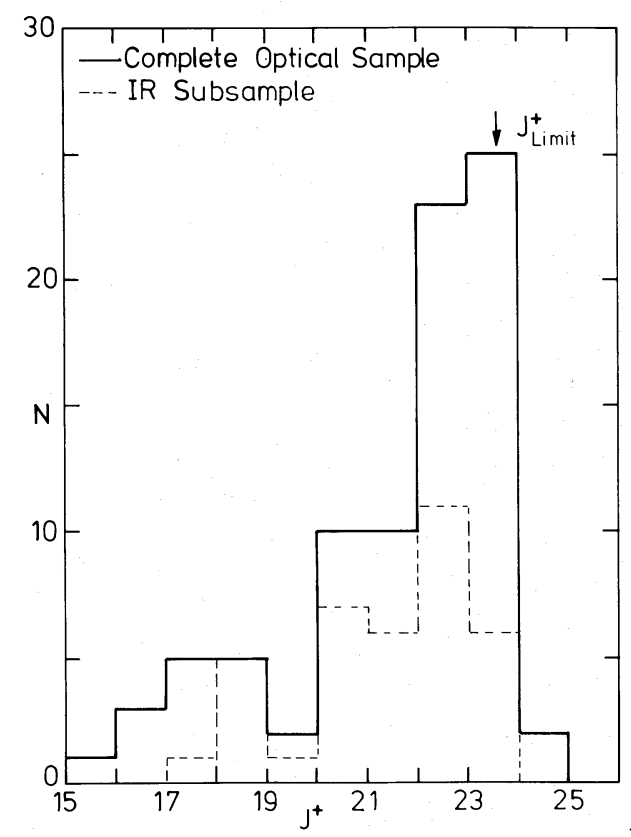

FIG. 1.-The $J^{+}$-magnitude distribution (uncorrected for galactic extinction) for the complete sample of Kron, Koo, and Windhorst (1984) without the Lynx field (86 galaxies; solid line) and for the present infrared sample without the Lynx field ( 37 galaxies; dashed line). The effective limit for complete optical identification $\left(\mathrm{J}^{+}=23.7 \mathrm{mag}\right)$ is shown by the arrow.

\section{ANALYSIS}

\section{a) Models}

To interpret our observations we shall use mainly the set of models for the spectral evolution of galaxies constructed by Bruzual (1981, 1983). Bruzual has convolved the results of his models with the appropriate filter responses in the Johnson (1966) $U B V J H K$ system and the $U^{+} J^{+} F N$ system.

We have compared the infrared color-magnitude tables computed by Bruzual (1981) with similar tables computed for the IRTF filter system. We found agreement to within 0.15 mag. This is also what is expected when the observationally determined color transformations between the Johnson (1966) and the IRTF systems are considered:

$$
\begin{aligned}
& (J-H)_{\mathrm{J}}=0.92(J-H)_{\mathrm{IRTF}} \text {, } \\
& ( \pm 0.01) \\
& (H-K)_{\mathrm{J}}=1.05(H-K)_{\mathrm{IRTF}} \text {. } \\
& ( \pm 0.02)
\end{aligned}
$$

Because the difference between the two systems is smaller, in most cases, than the observational errors, Bruzual's models have been used because they cover a wider range of parameters than ours.

We shall discuss primarily two types of models, which differ mainly by their star formation rate (SFR): the single-burst, or $\mathrm{C}$, models, which have a constant SFR during the first $10^{9} \mathrm{yr}$ and no star formation afterward, and the $\mu$ models, which are characterized by an exponentially decreasing SFR. The quantity $\mu$ represents the fraction of the original galaxy mass that would be in stars after $10^{9} \mathrm{yr}$ if dying stars did not return gas. Thus models with $\mu \gtrsim 0.5$ describe early-type (elliptical) galaxies where most of the star formation occurs early, while low- $\mu$ models $(0.01 \lesssim \mu \lesssim 0.2)$ give a better approximation to spiral galaxies that have star formation throughout their lifetimes. Models with low $\mu(<0.5)$ have kindly been made available by Dr. G. Bruzual. To compare our data with spiral galaxy models, we have also used nonevolving spiral models constructed from the data of Coleman, Wu, and Weedman (1980) with $K$-corrections calculated with the appropriate filter responses. Internal reddening was not included in any of the models.

In order to convert galaxy age to redshift, we need to specify the cosmological model. Throughout we adopt galaxy ages of $16 \times 10^{9} \mathrm{yr}$, with $H_{0}=50 \mathrm{~km} \mathrm{~s}^{-1} \mathrm{Mpc}^{-1}$ and $q_{0}=0$. We shall discuss how changing the values of $H_{0}$ and $q_{0}$ affects our conclusions in $\S$ III $d$. Since we do not have redshift information for all objects, we replace the redshift by apparent magnitude after specifying an absolute magnitude, and analyze our data in terms of color-color and color-apparent magnitude diagrams. Each curve in the color-magnitude diagrams is thus characterized by one absolute magnitude, with each point on the curves characterized by a redshift value. In order to illustrate the range of model predictions, we give in each figure two models, one with $M_{v}=-23$, characteristic of the most luminous galaxies, and one with $M_{v}=-20$, characteristic of relatively low luminosity galaxies $\sim 2$ mag below the break in the optical luminosity function. Models with intermediate luminosity will lie between these two models.

\section{b) Infrared Color-Color Diagram}

We examine first the data in the infrared $(\mathrm{J}-\mathrm{H}, \mathrm{H}-\mathrm{K})$ color-color diagram (Fig. 2). Since this diagram involves only infrared colors, it gives mainly information on the old stellar 


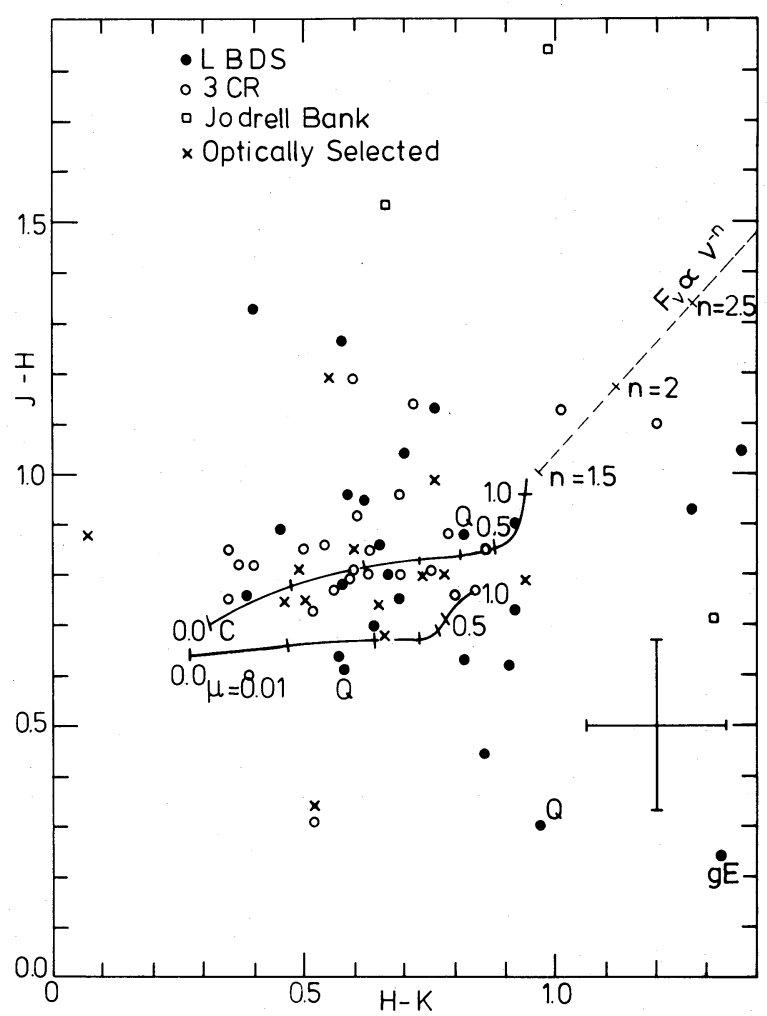

FIG. 2.-The infrared color-color diagram, corrected for galactic extinction. Four different samples are plotted, while the quasar points are marked $\mathrm{Q}$. The model drawn is from Bruzual (1981) for $H_{0}=50 \mathrm{~km} \mathrm{~s}^{-1} \mathrm{Mpc}^{-1}, q_{0}=0$, and galaxy age $=16 \times 10^{9} \mathrm{yr}$. Redshift intervals of 0.1 are marked on the model line. The error bars represent the mean of all observed Leiden-Berkeley galaxies. The dashed line is the power-law locus with various spectral indices.

populations in the observed galaxies and on possible nonthermal contributions, which may show up by an infrared excess at $2.2 \mu \mathrm{m}$. Along with the LBDS sources (filled circles), we have also plotted the 3CR radio sources (open circles) observed by Lilly and Longair (1982), the optically selected galaxies of Ellis and Allen (1983) (crosses), and the steepspectrum compact (Jodrell Bank) radio sources observed by Lebofsky, Rieke, and Walsh (1983) (open squares). The same symbols are used for these surveys in all figures. The $\mathrm{C}$ model with $M_{v}=-23 \mathrm{mag}$ is superposed on the data points. High- $\mu$ models $\left(0.5 \lesssim \mu \lesssim 1.0\right.$ and $\left.M_{v}=-23\right)$ are not distinguishable from the $\mathrm{C}$ model. Low $-\mu\left(\mu \sim 0.01\right.$ and $\left.M_{v}=-23\right)$ spiral models have bluer colors for a given redshift. Several conclusions can be obtained from Figure 2 . Since they concern only colors, these conclusions are to first order independent of the absolute magnitudes of the galaxies and, hence, of $H_{0}$ and $q_{0}$. Since detection in all three infrared bands could generally be done for LBDS galaxies with $K \leq 16 \mathrm{mag}$, the conclusions are valid only for galaxies in that magnitude range.

First, the infrared colors are independent of the radio flux, and hence radio power, of the galaxy. Although the LBDS and $3 \mathrm{CR}$ radio galaxies differ by more than 3 orders of magnitude in their radio power, they are indistinguishable in the $(J-H$, $H-K)$-plane. This conclusion can be extrapolated to very low radio powers, since the bright $(K \lesssim 16 \mathrm{mag})$ optically selected galaxies (thus, presumably without radio emission at the $\mathrm{mJy}$ level) of Ellis and Allen (1983) are also indistinguishable from the LBDS and 3CR radio sources in the $(J-H, H-K)$-plane. Thus, whatever causes the strength of the radio emission is unrelated to the old stellar populations in the radio galaxies. Since the majority of the $3 \mathrm{CR}$ radio sources are associated with giant elliptical galaxies, we conclude that the stellar populations in mJy radio sources with $K \lesssim 16$ mag are like those of elliptical galaxies, in agreement with the direct comparison of the data with Bruzual's models.

Second, the $\mathrm{C}$ or high- $\mu$ models for elliptical galaxies give a good fit to the bulk of the LBDS data, with the model line nearly bisecting the data points. The spiral model with $\mu=0.01$ clearly does not give a good fit to most of the points, although about one-third of the mJy radio galaxies may be explained better by a low- $\mu$ model than by a high- $\mu$ model. While the spread in colors of most points on either side of the $\mathrm{C}$-model line is consistent with the observational errors, the spread along the C-model line is due mainly to a redshift effect. The redshifts are marked on the model lines. The LBDS galaxies with $K \lesssim 16$ mag span a redshift range from $\sim 0.1$ to $\sim 0.75$. This range of redshifts implied by the observed infrared colors is entirely consistent with the range of spectroscopic redshifts obtained by Kron, Koo, and Windhorst (1984) for 14 galaxies in the infrared sample, listed in Table 1.

There are several data points that cannot be explained by either an elliptical or a spiral model. These are points with very red $(H-K)$ or $(J-H)$ colors. Neither $\mathrm{C}$ nor $\mu$ models can produce $(H-K)$ colors redder than $\sim 1.0$, which is shown, for example, by the turn-up of the $\mathrm{C}$-model line at $(H-K) \sim 0.9$ mag. This limit in the $(H-K)$ color may be an artifact of Bruzual's models, where for stellar masses smaller than $1.7 M_{\odot}$ the empirical local giant luminosity function of Tinsley and Gunn (1976) is used. The possible lack of self-consistency and universality of this local giant luminosity function, and the manner in which this function is introduced in the models, may artificially reduce the evolution in the infrared (Bruzual 1983). Nevertheless, the observations appear to be consistent with such a limit. There are only three LBDS galaxies (out of a total of 25$)$ that have $(H-K) \gtrsim 1.0 \mathrm{mag}$. Such a red $(H-K)$ color may be caused by nonthermal emission. The addition of a power-law spectrum (dashed line in Fig. 2) to an elliptical stellar population can probably reproduce the very red $(H-K)$ colors and elliptical-like $(J-H)$ colors. The three LBDS objects with very red $(J-H)[(J-H) \gtrsim 1.10]$ cannot be explained in the same manner because the $(H-K)$ color resulting from such a combination would be too red compared to those observed. Thuan (1984) has shown that such red $(J-H)$ colors in conjunction with elliptical-like $(H-K)$ colors can be explained by the addition of carbon stars to an elliptical stellar population. In any case, the infrared colors do not show a large fraction of objects with strong nonthermal or stellar infrared contributions in the LBDS sample. This conclusion will be confirmed when the optical and infrared data are combined $(\S \operatorname{III} d)$.

We note finally that none of the LBDS and 3CR radio sources have $(J-H)$ color as red as those of two of the three empty field steep-spectrum radio sources (open squares) observed by Lebofsky, Rieke, and Walsh (1983). The infrared fluxes of the latter sources surely have a large nonstellar contribution, which may be due to their selection as steep-spectrum, compact sources.

\section{c) Infrared Color-Magnitude Diagrams}

We now examine the infrared color-magnitude diagrams $(H-K, K)$ in Figure 3 and $(J-K, K)$ in Figure 4 . In each of the figures we have again plotted the four samples: the LBDS 


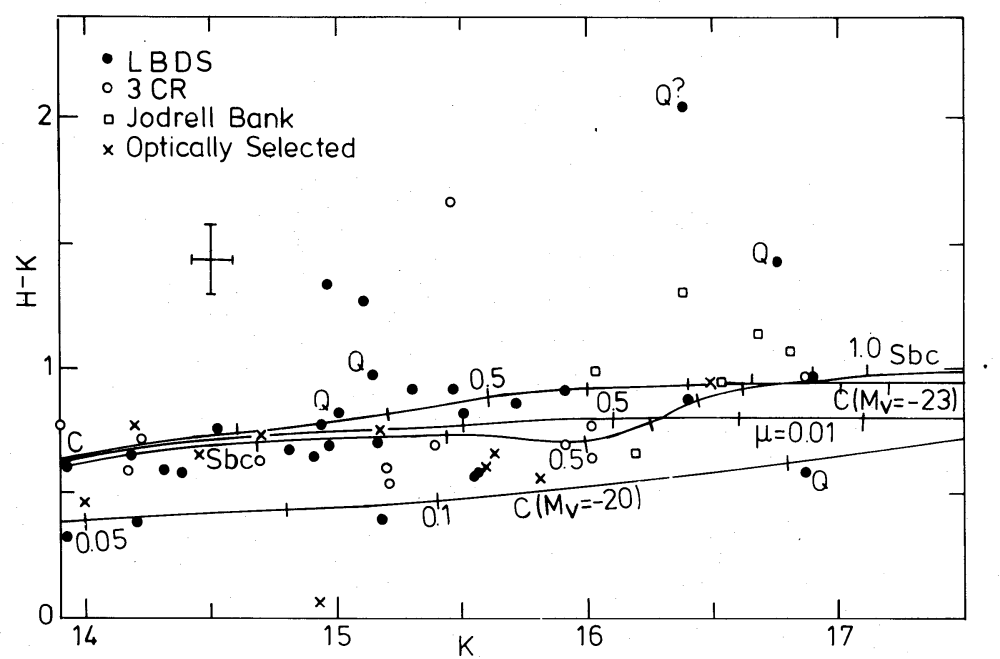

Fig. 3.-The $(H-K, K)$ infrared color-magnitude diagram. Superposed on the data points are the $\mathrm{C}$ and $\mu=0.01$ models by Bruzual (1981) and a nonevolving model for an Sbc galaxy derived from Coleman, Wu, and Weedman (1980). The top three models are for $M_{v}=-23$, andthe bottom model is for $M_{v}=-20$.

identifications, the $3 \mathrm{CR}$ radio galaxies, the optically selected galaxies, and the steep-spectrum compact radio sources of the Jodrell Bank survey. Superposed on the data points is the $\mathrm{C}$ model for two different absolute visual magnitudes $\left(M_{v}=-23\right.$ and $M_{v}=-20$ ). Also plotted is the prediction for a nonevolving late-type Sbc galaxy, as well as the model for an evolving spiral with $\mu=0.01$ and $M_{v}=-23$. Since nearinfrared colors are most sensitive to old stellar populations and not very sensitive to young stars, the early-type $\mathrm{C}$ model and the nonevolving Sbc model are very similar. The model with later star formation $(\mu=0.01)$ is not very different from the previous models in the $(H-K, K)$-plane, but begins to deviate from them in the $(J-K, K)$-plane, where the color baseline is longer.

Figures 3 and 4 show that a large fraction $(z 65 \%)$ of the LBDS and 3CR points can be represented by early-type $\mathrm{C}$ models or mildly evolving elliptical models with high $\mu(\mu \gtrsim$ $0.5)$ and $M_{v}=-23$. This conclusion was also reached by Lilly and Longair (1982) and Puschell, Owen, and Laing (1982) for the 3 CR sources. It appears now that a substantial fraction of the mJy radio sources also have an SED compatible with that of luminous $\left(M_{v} \sim-23\right)$ elliptical galaxies. The models predict a redshift range of $0.1 \lesssim z \lesssim 0.7$ for the observed mJy sources, which is in agreement with the range of measured redshifts (Table 1).

But there is also a nonnegligible fraction $(\sim 30 \%)$ of LBDS galaxies that have significantly bluer $(J-K)$ and $(H-K)$ colors or fainter $K$-magnitudes than that predicted by models for luminous elliptical galaxies $\left(M_{v} \sim-23\right)$. These points can be explained by combinations of models with lower $\mu$ and/or optical luminosities. For example, these points may be due to a population of luminous spiral galaxies. The $\mu=0.01$ model for $M_{v}=-23$ can provide a reasonable fit to most of the blue points. In this case, the blue colors are due mainly to late star formation. Alternatively, the blue points may represent galaxies with less active late star formation but with lower optical luminosities $\left(-22 \lesssim M_{v} \lesssim-20\right)$ and thus with lower redshifts $(z \lesssim 0.3)$. The colors then appear bluer because they are not redshifted as much. Figures 3 and 4 show, for example, that the C model with $M_{v}=-20$ can also give a good fit to the blue

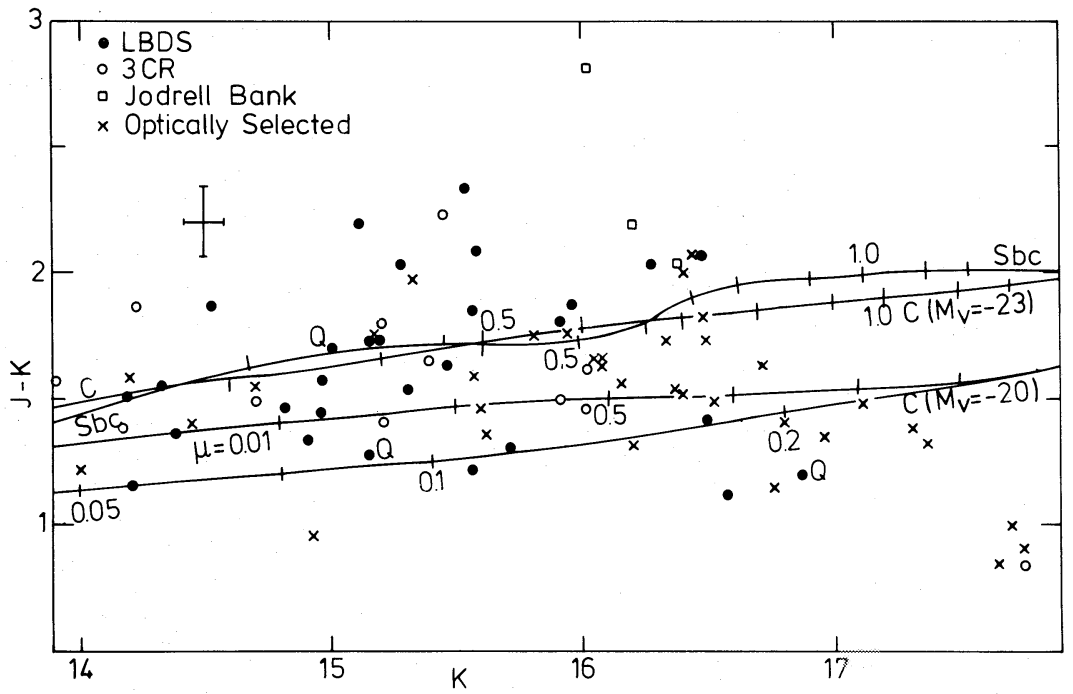

Fig. 4.-The $(J-K, K)$ infrared color-magnitude diagram. The same comments as in Fig. 3 apply. 
points. Of course, any combination of these two effects can also explain the blue points.

Only two blue $[(J-K) \lesssim 1.20 \mathrm{mag}]$ galaxies in Table 1 possess both infrared color measurements and measured redshifts: 53W 020 has a redshift of 0.10 and $(J-K)=1.15$, and 53W 076 has a redshift of 0.39 and $(J-K)=1.21$. These two redshift measurements would tend to favor the interpretation that the blue galaxies are low-redshift and low-luminosity starforming galaxies. We shall see that this conclusion is reinforced when the optical-infrared color-magnitude diagrams are analyzed $(\S \operatorname{III} d)$.

It is interesting to note that the $(J-K)$ and $(H-K)$ colors of the blue galaxies are similar to those of nearly blue compact galaxies where intense bursts of star formation are occurring (Thuan 1983), when $K$-corrections are taken into account. The steep-spectrum blank field radio sources are too red with respect to the models, suggesting again strong nonthermal infrared excesses in these sources. In Figure 3 the points with very red $(H-K)$ colors correspond to quasars with strong nonthermal contributions in the near-infrared.

The changes in the infrared colors and magnitudes as a function of $H_{0}$ and $q_{0}$ are very small. For example, $(J-K)$ is bluer by $0.01 \mathrm{mag}$ for a $\mathrm{C}$ model or a model with $\mu=0.70$, and the $K$-magnitudes are fainter by $0.02 \mathrm{mag}$ at $z=0.5$, when $H_{0}$ is changed from 50 to $100 \mathrm{~km} \mathrm{~s}^{-1} \mathrm{Mpc}^{-1}$ (Bruzual 1981). Changing $q_{0}$ from 0 to 0.5 would make $K$ brighter by 0.07 mag at $z=0.5$. Thus none of our preceding conclusions depend sensitively on the precise values of $H_{0}$ and $q_{0}$. Since internal reddening in galaxies is very small in the infrared $(\lesssim 0.03 \mathrm{mag}$ at $J$ and smaller at longer wavelengths), the neglect of internal reddening in the model galaxies cannot affect these conclusions.

\section{d) Optical-Infrared Color-Magnitude Diagrams}

We now combine our near-infrared measurements with the optical magnitudes of Kron, Koo, and Windhorst (1984). The longer baseline in wavelength will make it possible to dis- tinguish better the blue galaxy population already suggested by the infrared data.

We construct color-magnitude diagrams combining the infrared $K$-magnitude with the measured optical magnitudes $U^{+} J^{+} F N$ (Figs. 5-8) and the derived optical magnitudes $B V R$ (Figs. 9-11). Depending on the availability of optical magnitudes, the LBDS sample is plotted in all figures, the 3CR sample of Lilly and Longair (1982) has only $V$ - and $R$ magnitudes and is plotted in Figures 10 and 11, and the optically selected sample of Ellis and Allen (1983) with only $\mathrm{J}^{+}$-magnitudes available is plotted in Figure 6. The optical plate limit is shown by the oblique line in each figure.

Examination of Figures 5-11 reveals the following general features.

1. The LBDS data do not show evidence for a large population of very red galaxies. This statement holds for $K \lesssim 16$ mag, where the selection effects, due to the plate limits, against observing very red galaxies are not yet operative. Some objects ( $\lesssim 10 \%$ of the sample) with colors significantly redder than those given by the $\mathrm{C}$ model with $M_{v}=-23$ may be explained as having some nonthermal contribution or a population of carbon stars (Thuan 1984). The larger fraction of red objects found by Windhorst, Puschell, and Thuan (1983) was due to preliminary inaccurate optical magnitudes in the Hercules field.

2. About two-thirds of the mJy radio galaxies can be represented in all colors by the model predictions for nonevolving or mildly evolving $(\mu \gtrsim 0.5)$ luminous early-type galaxies with $M_{v}=-23$. Again the data suggest a substantial population of giant elliptical galaxies among the LBDS radio sources.

The remaining galaxies (about one-third of the infrared sample) have too blue colors to be explained by a $\mu=0.5$, $M_{v}=-23$ bright elliptical model. This was suggested earlier by the infrared color-magnitude diagrams (§ III $c$ ) but is amplified here by the larger optical-infrared baseline. The blue galaxies are present in all color-magnitude diagrams. In the diagram with the longest color baseline $\left(U^{+}-K, K\right)($ Fig. 5)

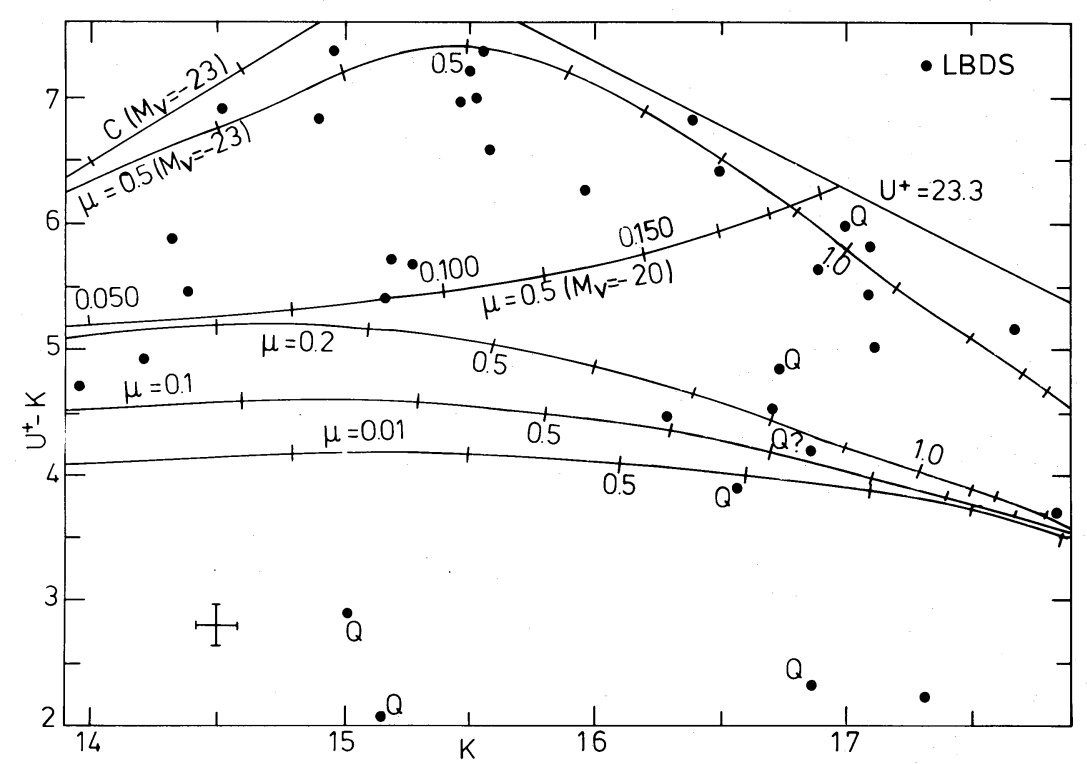

FIG. 5.-The $\left(U^{+}-K, K\right)$ optical-infrared color-magnitude diagram. Only the Leiden-Berkeley sample possesses $U^{+}$photometry. The oblique straight line on the top right-hand side of the figure represents the $U^{+}$plate limit. Bruzual's $(1981,1983) \mathrm{C}$ and $\mu=0.5,0.2,0.1,0.01$ models have been plotted, as well as a low-luminosity model with $\mu=0.5$ and $M_{v}=-20$. For further details see comments in Fig. 2 . 


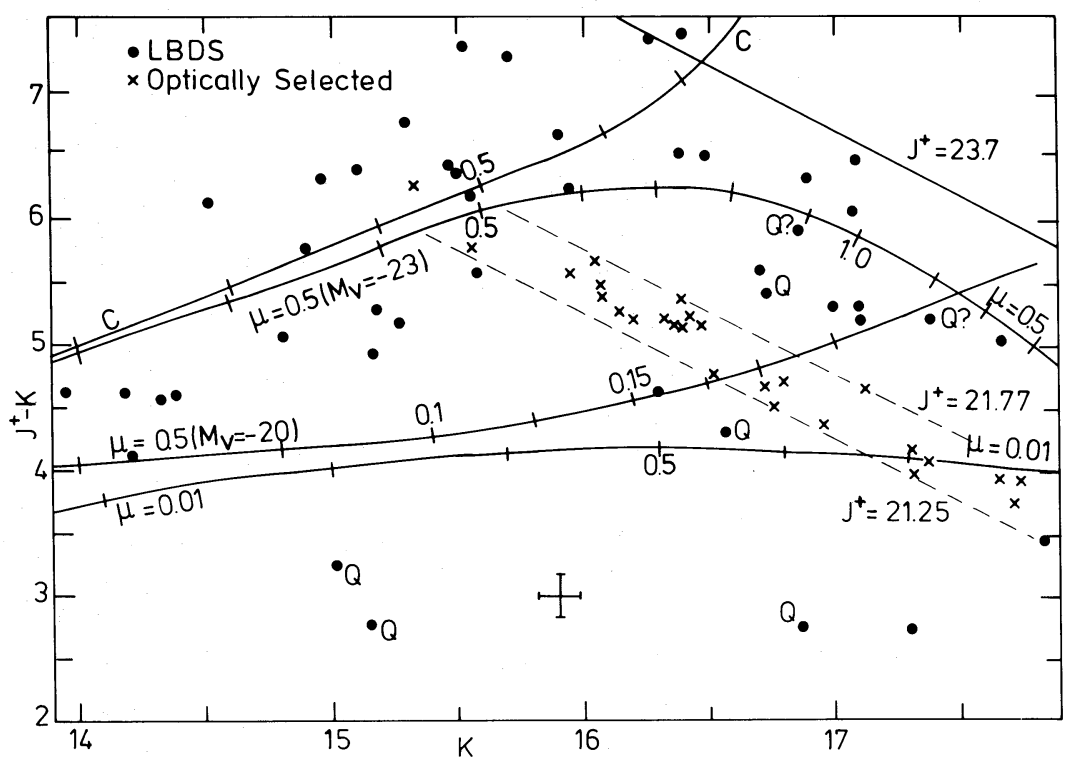

FIG. 6.-The $\left(J^{+}-K, K\right)$ optical-infrared color-magnitude diagram. Besides the Leiden-Berkeley sample, $J^{+}$photometry is also available for Ellis and Allen's (1983) optically selected sample, which has $21.25 \leq J^{+} \leq 21.77$. For further details see comments in Fig. 5 .

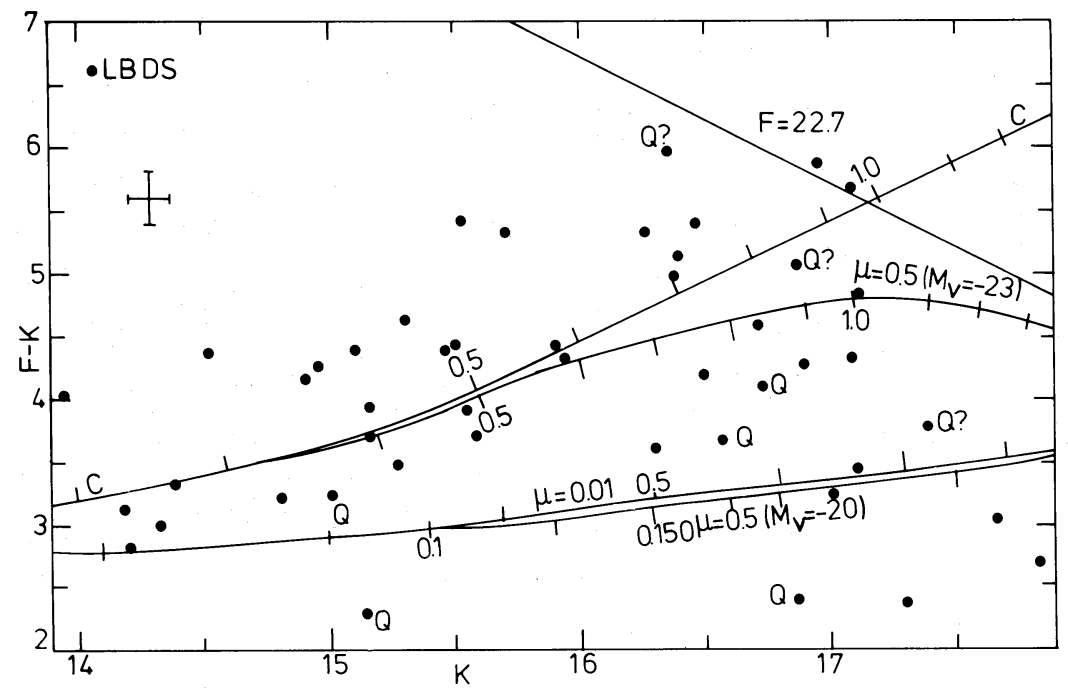

Fig. 7.-The $(F-K, K)$ optical-infrared color-magnitude diagram. For further details see comments in Fig. 5.

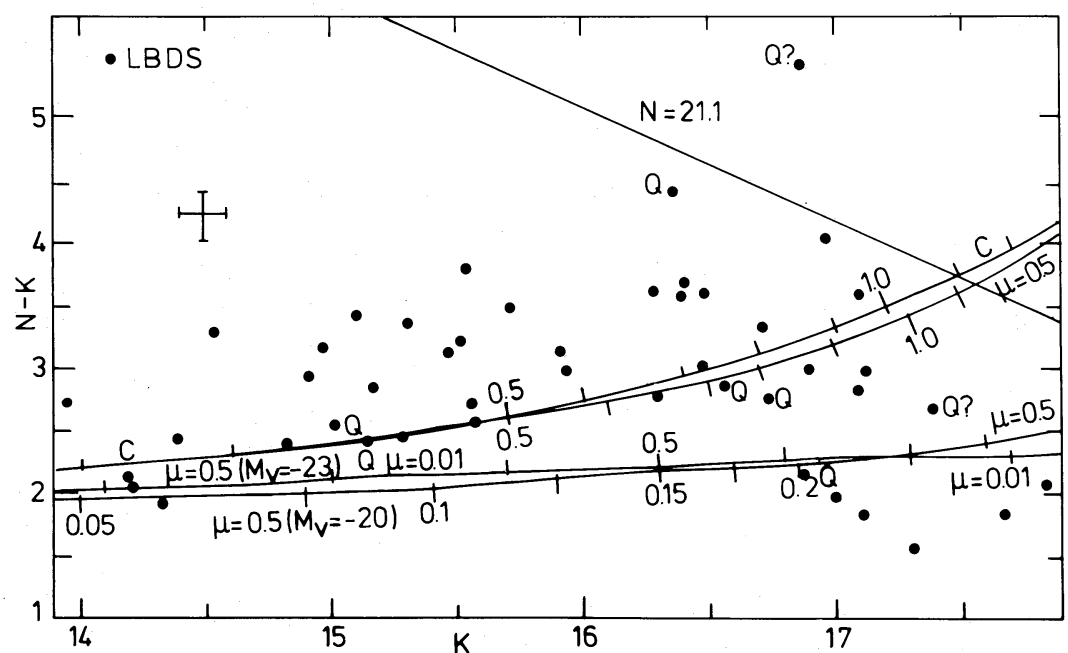

Fig. 8.-The $(N-K, K)$ optical-infrared color-magnitude diagram. For further details see comments in Fig. 5. 


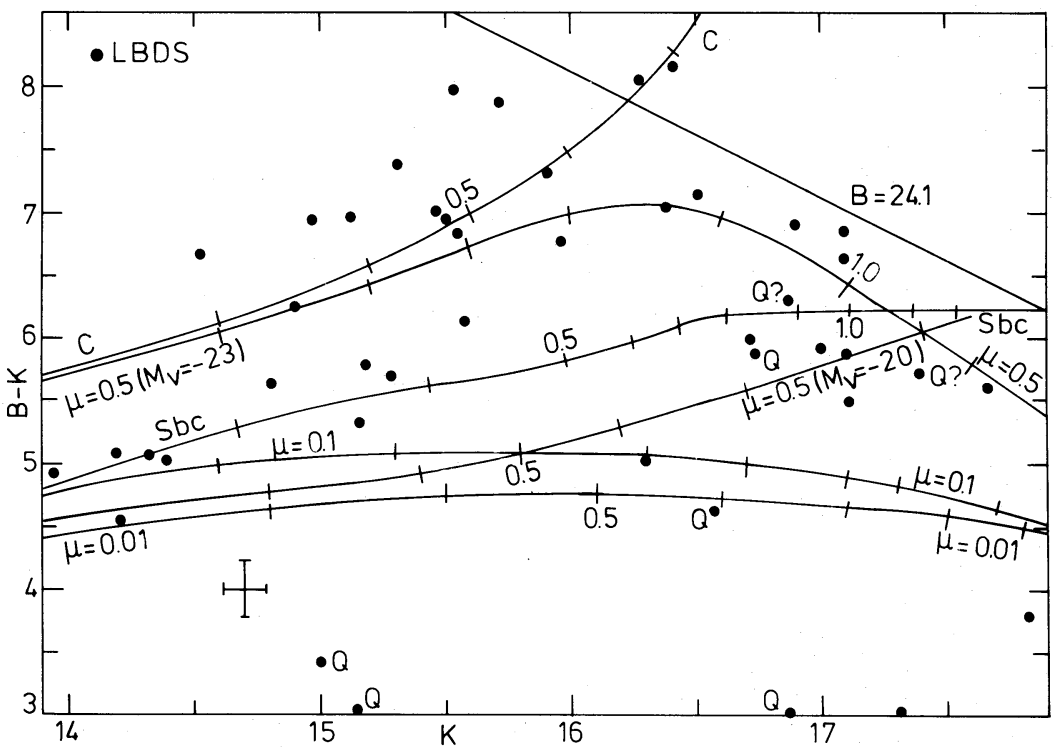

FIG. 9.-The $(B-K, K)$ optical-infrared color-magnitude diagram. Besides the high-luminosity $\left(M_{v}=-23\right) \mathrm{C}$ and $\mu=0.5,0.1,0.01$ models and the lowluminosity $\left(M_{v}=-20\right) \mu=0.5$ model, the nonevolving Sbc model is also plotted. For further details see comments in Fig. 5.

they are already present at $K \sim 14$ mag but are evident for $K \gtrsim 16.5 \mathrm{mag}$ only for shorter color-baseline diagrams (see Figs. 7 and 8). As already outlined in $\S$ III $c$, the bluer galaxies can be explained in two ways. They can be luminous $\left(M_{v} \sim\right.$ $-23)$ and distant $(0.3 \lesssim z \lesssim 0.7)$ galaxies with active star formation. Low $-\mu(0.01 \lesssim \mu \lesssim 0.2)$ models with $M_{v}=-23$ could reproduce the observed colors of most blue galaxies. Alternatively, the blue galaxies can be understood as nearer $(z \lesssim 0.3)$ galaxies of lower luminosity $\left(M_{v} \gtrsim-22\right)$, with less active star formation $(0.01 \lesssim \mu \lesssim 0.7)$. In the $(F-K, K)$ - and $(N-K, K)$ planes, for example (Figs. 7 and 8), the model with $\mu=0.5$ and $M_{v}=-20$ is practically indistinguishable from the model with $\mu=0.01$ and $M_{v}=-23$.
The latter explanation appears to be supported by the available redshift information. There are 11 galaxies with both redshift and optical-infrared colors, and in particular with the longest baseline color, $U^{+}-K$ (Table 1). Of these, four galaxies have blue $\left(U^{+}-K\right)$ colors $\left[\left(U^{+}-K\right)<5.5\right]$ : 52W 023 with $\left(U^{+}-K\right)=2.24$ and $z=0.202,53 \mathrm{~W} 010$ with $\left(U^{+}-K\right)=4.91$ and $z=0.100,54 \mathrm{~W} 013$ with $\left(U^{+}-K\right)=4.71$ and $z=0.249$, and $54 \mathrm{~W} 028$ with $\left(U^{+}-K\right)=5.47$ and $z=0.173$. All redshifts are less than 0.25 , suggesting that the blue galaxies are lowredshift and relatively low-luminosity star-forming galaxies. As suggested by the similarity in the infrared colors $(\S$ III $c)$, the mode of star formation in these galaxies may be similar to that in the nearby blue compact galaxies, where star formation

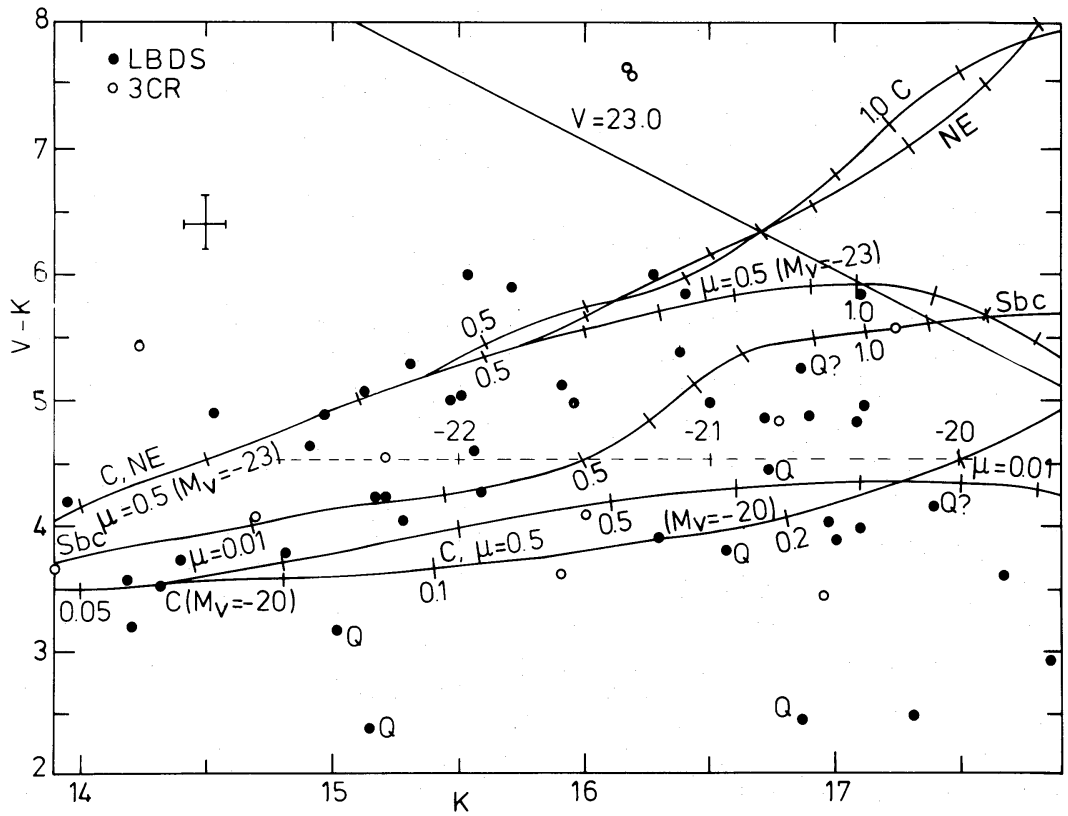

FIG. 10.-The $(V-K, K)$ optical-infrared color-magnitude diagram. Besides the Leiden-Berkeley sample, the 3CR sample of Lilly and Longair (1982) has also been plotted. The dashed horizontal line shows how the apparent $K$-magnitude changes as a function of absolute visual magnitude (see labels on dashed line) at a given redshift and color. For further details see comments in Fig. 9. 


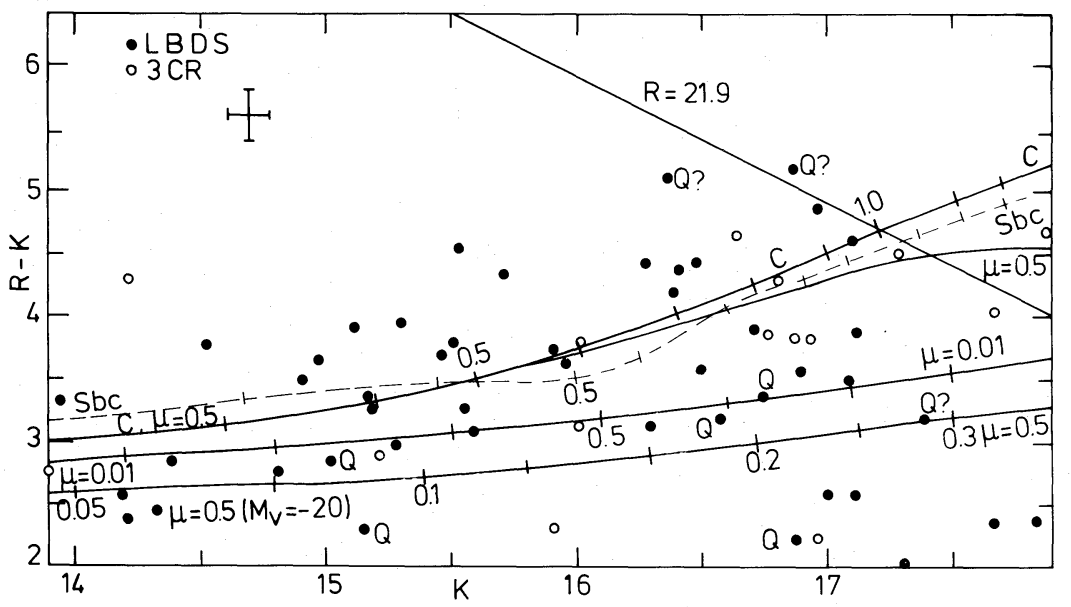

Fig. 11.-The $(R-K, K)$ optical-infrared color-magnitude diagram. For further details see comments in Fig. 10.

occurs in intermittent bursts lasting only a few million years (Thuan 1983). The presence of radio emission suggests that the bursts were relatively recent $\left(\lesssim 10^{6} \mathrm{yr}\right.$ ).

Most of the galaxies in the optically selected sample $(21.25$ mag $<J^{+}<21.75 \mathrm{mag}$ ) of Ellis and Allen (1983) also have colors bluer than those predicted by the $\mu=0.5, M_{v}=-23$ model (Fig. 6). These galaxies can again be best explained by a population of lower-luminosity $\left(M_{v} \gtrsim-22\right)$ nearby $(z \lesssim 0.3)$ star-forming galaxies. The absence of radio emission suggests that the star formation bursts in these galaxies are relatively older.

3. In Figures 5-11 three quasars have very blue opticalinfrared colors, suggesting a nonthermal component in the UV and optical parts of their spectrum. The five others have redder colors, three of which have an uncertain classification and can be high-redshift elliptical galaxies. The other two quasars, one of them in a cluster, have neutral colors.

We now discuss briefly how the preceding results are affected by adopting other values of $H_{0}$ and $q_{0}$ for the models. The effect of $q_{0}$ is slight. The $K$-magnitudes are brightened by 0.07 mag at $z=0.5$ when $q_{0}$ changes from 0 to 0.5 . The effect of changing $H_{0}$ is more important. The longest baseline color $\left(U^{+}-K\right)$ (Fig. 5) becomes bluer by $1.65 \mathrm{mag}$ when $H_{0}$ changes from 50 to $100 \mathrm{~km} \mathrm{~s}^{-1} \mathrm{Mpc}^{-1}$, for $q_{0}=0$ and $\mu=0.5$ at $z=0.5$.

This would mean that the $M_{v}=-23, \mu=0.5$ model would predict too blue colors for the reddest points in Figure 5, when $H_{0}$ is changed from 50 to $100 \mathrm{~km} \mathrm{~s}^{-1} \mathrm{Mpc}^{-1}$. But the $H_{0}=100 \mathrm{~km} \mathrm{~s}^{-1} \mathrm{Mpc}^{-1}$ model does not predict blue enough colors to account for the blue points. Thus our previous conclusions regarding the existerice of a nearby low-luminosity galaxy population still remain valid. In any case, we feel that a model with $H_{0}=100 \mathrm{~km} \mathrm{~s}^{-1} \mathrm{Mpc}^{-1}$ is not very probable because it would give galaxy ages of $9 \times 10^{9} \mathrm{yr}$, which are unacceptably low.

Including internal reddening in the galaxy models would redden the predicted colors. The longest baseline color $\left(U^{+}-K\right)$ would be reddened by $\sim 0.15 \mathrm{mag}$ (Johnson 1968), a small enough effect so that none of our conclusions are altered if internal reddening is included in the models.

\section{SUMMARY AND CONCLUSIONS}

We have obtained near-infrared measurements for 40 galaxies and 8 quasars drawn from the complete sample of
Leiden-Berkeley Deep Survey (LBDS) radio sources of Windhorst, Kron, and Koo (1984). All galaxies have radio fluxes at $1412 \mathrm{MHz}$ greater or equal to $0.6 \mathrm{mJy}$ and a peak radio signalto-noise ratio greater than or equal to 5 . The majority of the galaxies have radio fluxes in the $\mathrm{mJy}$ range and optical magnitudes derived from photometry on deep $U^{+} J^{+} F N$ Mayall $4 \mathrm{~m}$ prime-focus plates (Kron, Koo, and Windhorst 1984). We have constructed infrared and optical-infrared color-color and color-magnitude diagrams and used the spectral evolution models of Bruzual $(1981,1983)$ to interpret our observations. We have compared our measurements with those obtained for the 3CR sample by Lilly and Longair (1982) and for an optically selected sample by Ellis and Allen (1983) and arrived at the following conclusions:

1. The mJy LBDS radio galaxies, the strong 3CR radio galaxies, and the optically selected (radio-quiet) field galaxies cannot be distinguished by their infrared and optical-infrared colors. The colors are independent of the radio flux and radio power of the galaxies and are probably due to their stellar populations.

2. The LBDS sample does not show evidence for a significant population of very red galaxies $(\lesssim 10 \%)$, implying that there is no significant population of objects with nonthermal $2.2 \mu \mathrm{m}$ excesses at faint magnitudes.

3 . The infrared and optical-infrared colors of about twothirds of the LBDS galaxies can be reproduced by a nonevolving or mildly evolving $(\mu \gtrsim 0.5)$ luminous giant elliptical galaxy model spectrum with $M_{v}=-23\left(H_{0}=50 \mathrm{~km} \mathrm{~s}^{-1}\right.$ $\mathrm{Mpc}^{-1}$ and $\left.q_{0}=0\right)$. The remaining third of the LBDS galaxies have colors bluer than those given by the luminous elliptical model. The available redshift information appears to indicate that the blue galaxies are nearer $(z \lesssim 0.3)$, lower-luminosity $\left(M_{v} \gtrsim-22\right)$ galaxies undergoing star formation $(0.01 \lesssim \mu \lesssim$ $0.7)$.

We thank the directors of the IRTF and the UKIRT for generous allotments of observing time and the staff at these telescopes for helpful and efficient assistance. We are grateful to Drs. Richard Kron and David Koo, who kindly communicated the optical magnitudes of the Leiden-Berkeley radio sources in advance of publication, and to Dr. Gustavo Bruzual, who kindly provided his small- $\mu$ models.

T.X.T. acknowledges partial financial support for NSF grant AST 81-19683, and thanks Dr. Jean Audouze for his hospital- 
ity at the Institut d'Astrophysique de Paris of the Centre National de la Recherche Scientifique, where part of this paper was written. R.A.W. acknowledges financial support by the Netherlands Organization for the Advancement of Pure Research ZWO (ASTRON grant 19-23-009 and a ZWO/
ASTRON travel grant). He thanks Harry van der Laan and Peter Katgert for their continuous encouragement of the project. We thank Sjaak Ober for drawing many crowded figures and Wim Brokaar for photographing them.
Bruzual, G. A. 1981, Ph.D. thesis, University of California, Berkeley. - 1983, Ap.J., 273, 105

Butcher, H. R., and Oemler, A. 1978, Ap. J., 219, 18

Coleman, G. D., Wu, C. C., and Weedman, D. W. 1980, Ap. J. Suppl., 43, 393.

Dressler, A., and Gunn, J. E. 1983, Ap. J., 270, 7.

Elias, J. H., Frogel, J. A., Matthews, K., and Neugebauer, G. 1982, A.J., 87, 1029.

Ellis, R. S., and Allen, D. A. 1983, M.N.R.A.S., 203, 685.

Johnson, H. L. 1966, Ann. Rev. Astr. Ap., 4, 193.

. 1968, in Stars and Stellar Systems, Vol. 7, Nebulae and Interstellar

Matter, ed. B. M. Middlehurst and L. H. Aller (Chicago: University of Chicago Press) p. 167.

Koo, D. C. 1981, Ph.D. thesis, University of California, Berkeley.

Kron, R. G. 1980, Ap. J. Suppl., 43, 305.

Kron, R. G., Koo, D. C., and Windhorst, R. A. 1984, Astr. Ap., in press.

Lebofsky, M. J. 1981, Ap. J. (Letters), 245, L59.

\section{REFERENCES}

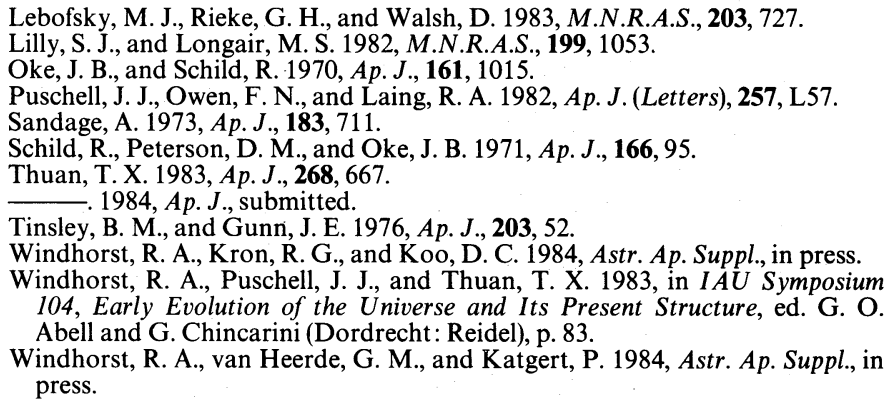

Schild, R., Peterson, D. M., and Oke, J. B. 1971, Ap. J., 166, 95.

Thuan, T. X. 1983, Ap. J., 268, 667 . 1984, Ap. J., submitted.

Tinsley, B. M., and Gunn, J. E. 1976, Ap. J., 203, 52.

Windhorst, R. A., Kron, R. G., and Koo, D. C. 1984, Astr. Ap. Suppl., in press.

Windhorst, R. A., Puschell, J. J., and Thuan, T. X. 1983, in IAU Symposium 104, Early Evolution of the Universe and Its Present Structure, ed. G. O. Abell and G. Chincarini (Dordrecht: Reidel), p. 83.

Windhorst, R. A., van Heerde, G. M., and Katgert, P. 1984, Astr. Ap. Suppl., in press.

RICHARD B. ISAACMAN: United Kingdom Infrared Telescope, 900 Leilani Street, Hilo, HI 96720

Frazer N. Owen: National Radio Astronomy Observatory, 1000 Bullock Boulevard, N.W., Socorro, NM 87801

JefFery J. PusChell: Titan Systems Inc., P.O. Box 12139, La Jolla, CA 92037

Trinh X. Thuan: Astronomy Department, University of Virginia, P.O. Box 3818, University Station, Charlottesville, VA 22903

Rogier A. Windhorst: Mount Wilson and Las Campanas Observatories, 83 Santa Barbara Street, Pasadena, CA 91101 